\title{
Dispersion and release of embelin from electrospun, biodegradable, polymeric membranes
}

\author{
Pablo R Cortez Tornello ${ }^{1,2}$, Gabriela E Feresin ${ }^{2}$, Alejandro Tapia ${ }^{2}$, Itiara G Veiga ${ }^{3}$, Ângela M Moraes ${ }^{3}$, \\ Gustavo A Abraham ${ }^{1}$ and Teresita R Cuadrado ${ }^{1}$
}

In this work, microfiber meshes containing embelin, a poorly water-soluble bioactive agent, were prepared by solubilizing embelin in a biodegradable and biocompatible polymer matrix of poly(e-caprolactone) (PCL). Plain or drug-loaded, highly porous, fibrous membranes with a high area-to-volume ratio were obtained by electrospinning. Non-woven microfibrous meshes were formed by uniform bead-free fibers with a mean diameter of $1.2 \mu \mathrm{m}$. Non-porous films were obtained by solution casting, and were used for comparison. The drug-loading content of the prepared systems was appropriate for topical applications. The thermal properties revealed that the crystallinity of embelin significantly decreased, the drug having almost completely dissolved in the PCL fibers. The in situ bioavailability of embelin, an antimycotic agent, is an important aspect to consider in topical drug applications. The drug-loaded systems presented different contact areas with the biological environment. When comparing the ability to expose embelin with the biological environment of the prepared systems, drug-loaded fibrous scaffolds showed a higher bioavailability of the bioactive agent because of an increase by $86 \%$ in the area-to-volume ratio, providing an effective area per unit mass that was 5.8 -fold higher than that of the film. For the meshes, $90 \%$ embelin release was observed after $12 \mathrm{~h}$ of exposure to phosphate-buffered saline, whereas for the films a comparable level of release occurred only after $72 \mathrm{~h}$.

Polymer Journal (2012) 44, 1105-1111; doi:10.1038/pj.2012.80; published online 16 May 2012

Keywords: biomaterials; drug dispersion; electrospinning; embelin; poly(e-caprolactone)

\section{INTRODUCTION}

There is a great deal of interest in designing devices to increase the bioavailability of poorly water-soluble drugs, which have a proven pharmacological activity for the treatment of several skin diseases. To achieve therapeutically important concentrations and to be optimally bioavailable to the skin, a drug must be in solution and in a nonionized state. In the case of topical delivery of poorly water-soluble or water-insoluble drugs, the solubilization of the drug in a matrix may be considered through the use of solid solution/dispersion technologies. $^{1,2}$ Although this technique is in its infancy with regard to pharmaceutical applications, a number of publications have suggested that the technique might be of a high value in topical drug administration and wound healing. ${ }^{3,4}$

The use of polymer-based, electrospun micro/nanofibers containing dispersions of poorly water-soluble pharmaceuticals has been reported for potential use in oral and topical drug delivery. ${ }^{3}$ Micro/ nanofibrous meshes have highly attractive functional characteristics, such as high surface area-to-volume ratios, a porous structure and biomimetic features. Because of these properties, non-woven materials offer interesting advantages for designing drug delivery systems. ${ }^{5-8}$ The high surface area of both the drug and the polymer carrier increases the dissolution rate of a particulate drug, and aids in mass transfer and efficient drug release. Depending on the polymer carrier and its geometry, the release kinetics from a pharmaceutical dosage form can be designed for rapid, immediate, delayed or modified dissolution.

Processing of polymer nanofibers through electrospinning has gained much attention in the last decade owing to its versatility for producing a wide variety of polymeric fibers and its ability to produce fibers in the submicron range, which is otherwise difficult to achieve with conventional, fiber-spinning technologies. ${ }^{6,9,10}$ To form micro/ nanofibers, a stream of a polymer solution or melt is subjected to a high electric field, forming a suspended droplet at the tip of a nozzle. The polymer jet is initiated when the electrostatic charge overcomes the surface tension of the droplet. Micro/nanofibers form when the ejection jet stream is narrowed under increasing surface charge density caused by solvent evaporation. A grounded target is used to collect the fibers, forming a non-woven mat. In the case of

\footnotetext{
${ }^{1}$ Instituto de Investigaciones en Ciencia y Tecnología de Materiales, INTEMA (UNMdP-CONICET), Mar del Plata, Argentina; ${ }^{2}$ Instituto de Biotecnología-Departamento de Electrónica y Automática. Facultad de Ingeniería, Universidad Nacional de San Juan, San Juan, Argentina and ${ }^{3}$ Departamento de Engenharia de Materiais e de Bioprocessos, Faculdade de Engenharia Química, Universidade de Campinas, Campinas, São Paulo, Brazil

Correspondence: Dr GA Abraham, Instituto de Investigaciones en Ciencia y Tecnología de Materiales, INTEMA (UNMdP-CONICET), Av. Juan B. Justo 4302, Mar del Plata, Buenos Aires B7608FDQ, Argentina.

E-mail: gabraham@fi.mdp.edu.ar
}

Received 20 December 2011; revised 9 March 2012; accepted 23 March 2012; published online 16 May 2012 
drug-loaded systems, the drug can be incorporated along with the polymeric solution to be electrospun. Fast and efficient solvent evaporation provides a limited time for the drug to recrystallize, which favors the formation of amorphous dispersions or solid solutions. ${ }^{4}$ Moreover, electrospinning affords great flexibility in selecting materials (for example, degradable or non-degradable polymers, synthetic or natural polymers and composites), bioactive agents and drugs for drug-delivery applications. ${ }^{5}$

In this work, polymeric films and electrospun, microfibrous meshes of poly( $\varepsilon$-caprolactone) (PCL) containing embelin, a poorly watersoluble bioactive agent, were prepared and characterized. PCL is a biocompatible, linear, aliphatic polyester with a glass transition temperature of $-60{ }^{\circ} \mathrm{C}$ and a melting temperature between 59 and $64^{\circ} \mathrm{C}$. Owing to its hydrophobic character and semicrystalline structure, PCL exhibits a long degradation time under physiological conditions, on the order of 2 years. This is an attractive property that is attractive for long-term applications in drug delivery. ${ }^{11}$ PCL degrades by hydrolysis, and the speed of this process depends on the shape and size of devices, on the bioactive agents dispersed in the polymer and on the physiological characteristics of the biological environment.

Embelin is a natural benzoquinone isolated as the main secondary metabolite from species of the Myrsinaceae $e^{12,13}$ and Oxalidaceae ${ }^{14}$ families. Embelin shows a diversity of relevant biological activities, such as a chemopreventive effect against $N$-nitrosodiethylamin/ phenobarbital (DENA/PB)-induced hepatocarcinogenesis in Wistar rats, ${ }^{15}$ anti-fertility effects, ${ }^{16}$ in vitro cytotoxic activity against B16 and XC cell lines, ${ }^{17}$ and anticonvulsant, ${ }^{18}$ antidiabetic ${ }^{19}$ and antimicrobial activities. ${ }^{14}$ Patel et al. ${ }^{20}$ studied the formulation of embelin tablets containing different diluents and employing wet granulation and direct compression techniques. To the best of our knowledge, the incorporation of embelin into polymeric matrices without using diluents has not been previously reported in the literature. The prepared embelin-based systems, including polymeric films and microfibrous meshes, were characterized in terms of drug content, thermal properties and morphology.

\section{EXPERIMENTAL PROCEDURE}

\section{Materials}

Embelin (2,5-dihydroxy-3-undecil-1,4-benzoquinone) was obtained from Oxalis erythrorhiza Gillies ex Hooker et Arnott (Oxalidaceae) ${ }^{12,21}$ collected in the province of San Juan, Argentina, following the procedure described by Feresin et al. ${ }^{14}$ Figure 1 shows the chemical structure of embelin. Its empirical formula is $\mathrm{C}_{17} \mathrm{H}_{26} \mathrm{O}_{4}$, and its molecular weight is $294.39 \mathrm{~g} \mathrm{~mol}^{-1}$. Embelin consists of a yellow-orange compound that crystallizes in the form of golden yellow needles. It is poorly soluble in water but highly soluble in solvents such as dichloromethane, methanol, petroleum ether and chloroform. PCL, with a number-average molecular weight of $80 \mathrm{~kg} \mathrm{~mol}^{-1}$, dichloromethane and methanol were obtained from Aldrich Chemical Co. (St Louis, MO, USA) and used without further purification.

\section{Films and microfibrous meshes preparation}

PCL solutions (10 wt \%) were prepared by dissolving PCL pellets in a dichloromethane:methanol solvent mixture (50:50 by volume) under magnetic stirring. For the preparation of drug-loaded films and meshes, 5 and 18 wt \% of embelin with respect to PCL were added to the polymeric solution, respectively. These amounts of drug were selected to assure the minimum inhibitory concentration index of embelin in each type of sample. Feresin et al. ${ }^{14}$ reported that embelin was active against Trypanosoma cruzi trypomastigotes at a minimum inhibitory concentration of $100 \mu \mathrm{g} \mathrm{ml}^{-1}$. Recently, Radhakrishnan et al. ${ }^{22}$ reported the bactericidal activity of embelin against Gram-positive organisms and bacteriostatic against Gram-negative organisms, with a minimum inhibitory concentration value $<100 \mu \mathrm{g} \mathrm{ml}^{-1}$ On the basis of that report, the prepared samples contained a sufficient

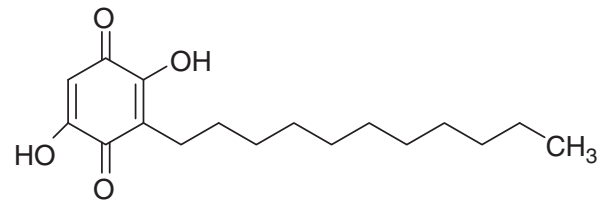

Figure 1 Chemical structure of embelin (2,5-dihydroxy-3-undecil-1,4benzoquinone).

amount of drug to act as effective drug-carrier systems for both bactericidal and trypanocidal activity. The flask in which the precursors were mixed was protected from light during the dissolution process, and a mixture was maintained under magnetic stirring at room temperature. The amount of embelin to be loaded was estimated by considering the amount required to prepare non-porous and porous discs containing $100 \mu \mathrm{g}$ of embelin.

Films were prepared by solution casting $(5 \mathrm{ml})$ onto a petridish $(4.6 \mathrm{~mm}$ in diameter). Drying was carried out under a fume hood at room temperature for $24 \mathrm{~h}$. Samples were subsequently vacuum dried to remove residual solvent. Electrospun microfibrous meshes were obtained by electrospinning. Each of the as-prepared solutions was loaded into a standard $10-\mathrm{ml}$ plastic syringe connected to a polyamide tube attached to the open end of a blunt, 18-gauge, stainless steel hypodermic needle used as a nozzle. The flow rate was controlled with a programmable syringe pump (Activa A22 ADOX S.A., Ituzaingó, Argentina). A high-voltage power source (ES30P, Gamma High Voltage Research Inc., Ormond Beach, FL, USA) was used to charge the solution by attaching the emitting electrode of positive polarity to the nozzle and the grounding electrode to the aluminum-collecting plate. All experiments were carried out at room temperature in a chamber having a ventilation system. The solutions (typically $5 \mathrm{ml}$ ) were electrospun at a positive high voltage of $17 \mathrm{kV}$, with a distance between the needle tip and the collecting plate of $12 \mathrm{~cm}$ and a solution-feeding rate of $0.6 \mathrm{ml} \mathrm{h}^{-1}$. The electrospun scaffolds were dried under vacuum at room temperature to fully eliminate the residual solvent and were stored in a desiccator.

Disc samples (3 $\mathrm{mm}$ in diameter) were cut and named $P C L_{\mathrm{f}}$ and $P C L_{\mathrm{m}}$, denoting the PCL films and PCL meshes, respectively, whereas the drug-loaded materials were referred to as $P C L E_{\mathrm{f}}$ and $P C L E_{\mathrm{m}}$ for embelin-loaded $P C L_{\mathrm{f}}$ and $P C L_{\mathrm{m}}$, respectively.

\section{Polymer and drug characterization}

Embelin content was determined by ultraviolet-visible spectroscopy (Agilent 8453, Santa Clara, CA, USA). Discs were dissolved in dichloromethane:methanol (50:50 by volume), and quantification was carried out after calibration, observing the absorption band at $\lambda=305 \mathrm{~nm}$. The loading efficiency was calculated from the ratio between the final and the initial embelin-to-polymer mass ratios. The thermal properties of PCL, embelin and embelin-loaded systems were determined by differential scanning calorimetry (Perkin Elmer Pyris 1, Waltham, MA, USA). Scans were performed at a heating rate of $5{ }^{\circ} \mathrm{C} \mathrm{min}^{-1}$. Thermograms were obtained under nitrogen purge. The degree of crystallinity of PCL $\left(X_{\mathrm{c}}\right)$ was calculated taking $148.05 \mathrm{Jg}^{-1}$ as the melting heat for pure high-molecular-weight PCL. ${ }^{23}$ The morphologies of the films and electrospun scaffolds were examined by scanning electron microscopy (JEOL JSM6460 LV, Peabody, MA, USA) after gold sputtering. The mean fiber diameter and diameter distribution were obtained with an image analyzer (Image-Pro Plus, Media Cybernetics, Inc., Bethesda, MD, USA).

\section{Embelin release measurements}

$P C L E_{\mathrm{f}}$ and PCLE $E_{\mathrm{m}}$ release behaviors (10 discs of $10 \mathrm{~mm}$ in diameter) were measured in glass flasks containing $200 \mathrm{ml}$ of phosphate-buffered saline at $\mathrm{pH}=7.4$ and $37^{\circ} \mathrm{C}$. Stirring was performed with an orbital shaker at 150 r.p.m.. Samples of $1 \mathrm{ml}$ were taken from the dissolution medium each $1 \mathrm{~h}$ during the first $12 \mathrm{~h}$, and one sample per day was taken during 18 days. A total of 30 extractions were performed. After each extraction, $1 \mathrm{ml}$ of fresh solvent was added. The total volume extracted was $<15 \%$ of the solution volume. All the assays were performed in triplicate. The concentration of embelin was determined by ultraviolet-visible spectroscopy (Agilent 8453) observing the absorption band at $\lambda=305 \mathrm{~nm}$. The percentage of embelin 
released was calculated by dividing the accumulated amount of embelin released at each sampling time by the initial amount of embelin $\left(m_{\mathrm{Emb}}\right)$.

\section{RESULTS AND DISCUSSION}

\section{Characterization of films and microfibrous meshes}

In electrospinning, the diameter and morphology of the produced micro/nanofibers are governed by a number of factors, including the solution parameters (solution dielectric constant, conductivity, polymer type and concentration and surface tension), the processing parameters (flow rate of the solution, applied voltage and needle tipto-collector distance) and the environmental parameters (temperature and humidity). ${ }^{24}$ The spinning conditions used in this work were selected based on a series of screening experiments to avoid electrospraying of the solution. Thus, the experimental parameters that were established led to the production of uniform and continuous fibers.

To study the surface morphology of electrospun meshes and films, the surfaces of the samples in contact with air, aluminum foil and glass were observed by scanning electron microscopy. No significant differences were found in the surfaces contacting air and either aluminum foil or glass. The typical appearance of the films and meshes obtained are shown in Figures 2-5.

The surface of $P C L_{\mathrm{f}}$ appeared to be smooth without visible pores, whereas $P C L_{\mathrm{m}}$ displayed a typical electrospun structure of random microfibers (Figures 2 and 4 ). The mesh exhibited a very high surface area-to-volume ratio, high porosity and an interconnected pore structure. $P C L_{\mathrm{m}}$ samples exhibited uniform bead-free fibers. According to a digital image-processing technique recently proposed by Gonzalez et $a .^{25}$ that allows the characterization of different morphological characteristics of electrospun scaffolds from scanning electron micrographs, the mean diameter of the fibers and their size distribution were determined. Figure 6 shows the fiber-diameter frequency distribution. The average fiber diameters were $1.267 \pm 0.576 \mu \mathrm{m}$ for $P C L_{\mathrm{m}}$ and $1.286 \pm 0.667 \mu \mathrm{m}$ for $P C L E_{\mathrm{m}}$, which indicates that the amount of embelin incorporated in the polymeric solution did not cause a significant change in the mean fiber diameter and morphology.

As shown in Figure 3, PCLE $E_{\mathrm{f}}$ micrographs reveal needle-like structures that can be ascribed to the presence of a small fraction of crystallized embelin. The same feature is observed in the $P C L E_{\mathrm{m}}$

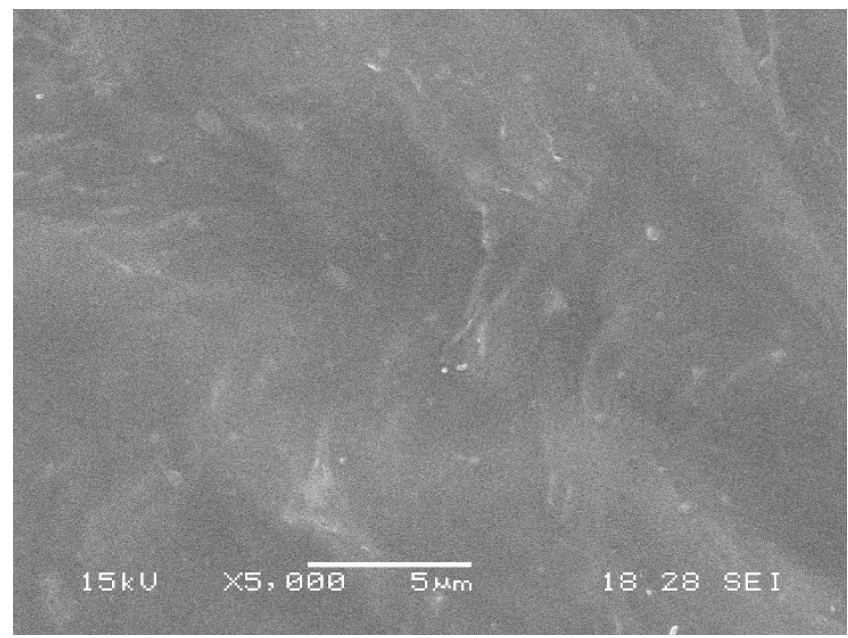

Figure 2 Scanning electron micrograph of $P C L_{f}$ (surface exposed to air; $\times 5000$ ).

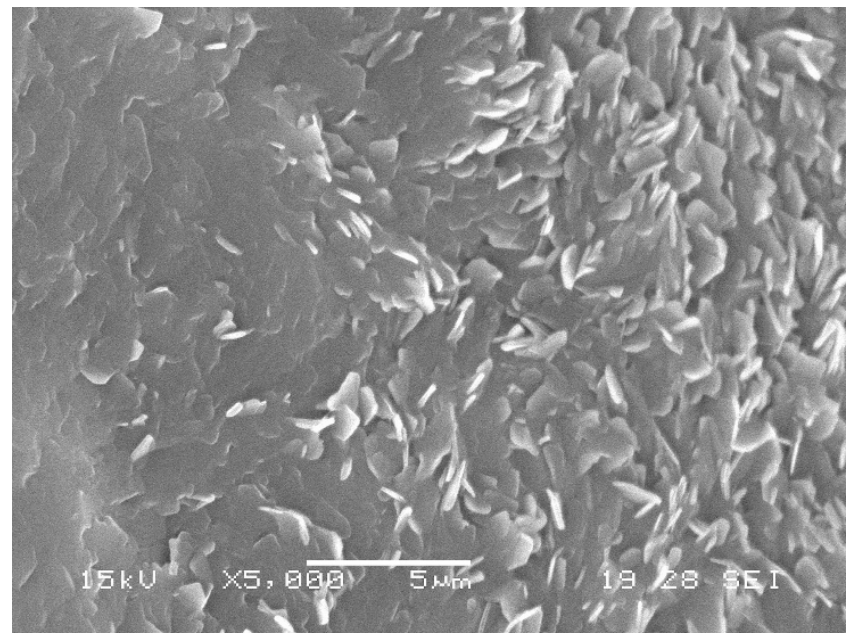

Figure 3 Scanning electron micrograph of $P C L E_{f}$ (surface exposed to air; $\times 5000$ ).

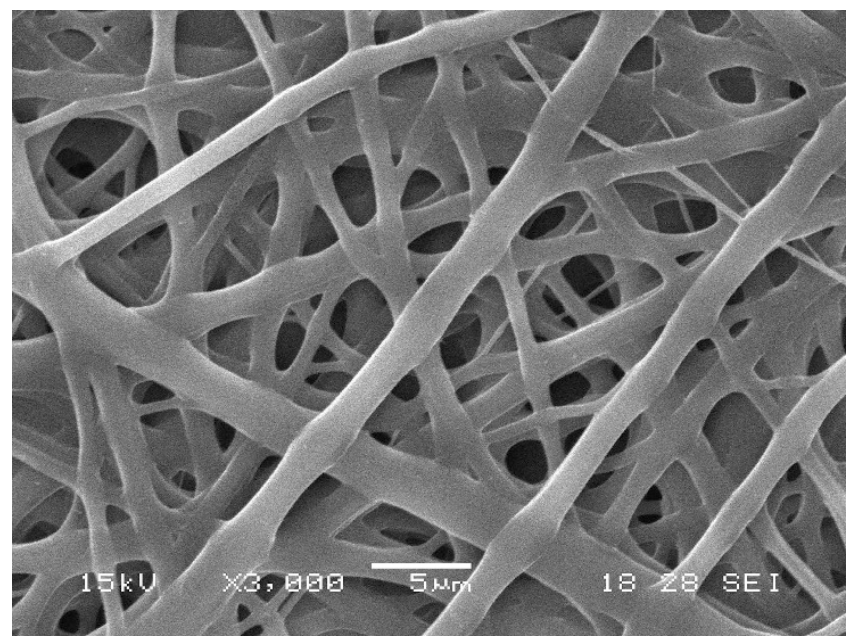

Figure 4 Scanning electron micrograph of $P C L_{m}$ (surface exposed to air; $\times 3000)$.

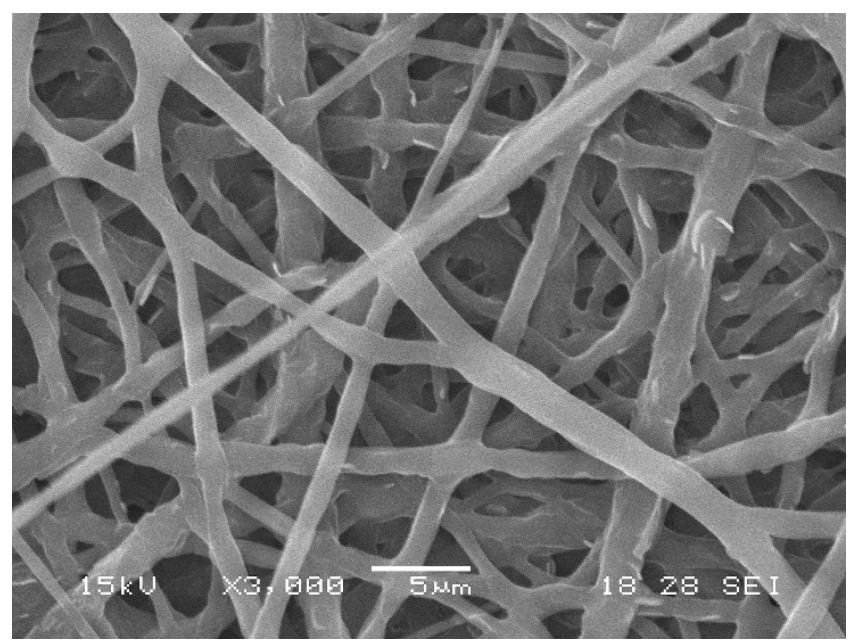

Figure 5 Scanning electron micrograph of $P C L E_{\mathrm{m}}$ (surface exposed to air; $\times 3000$ ). 

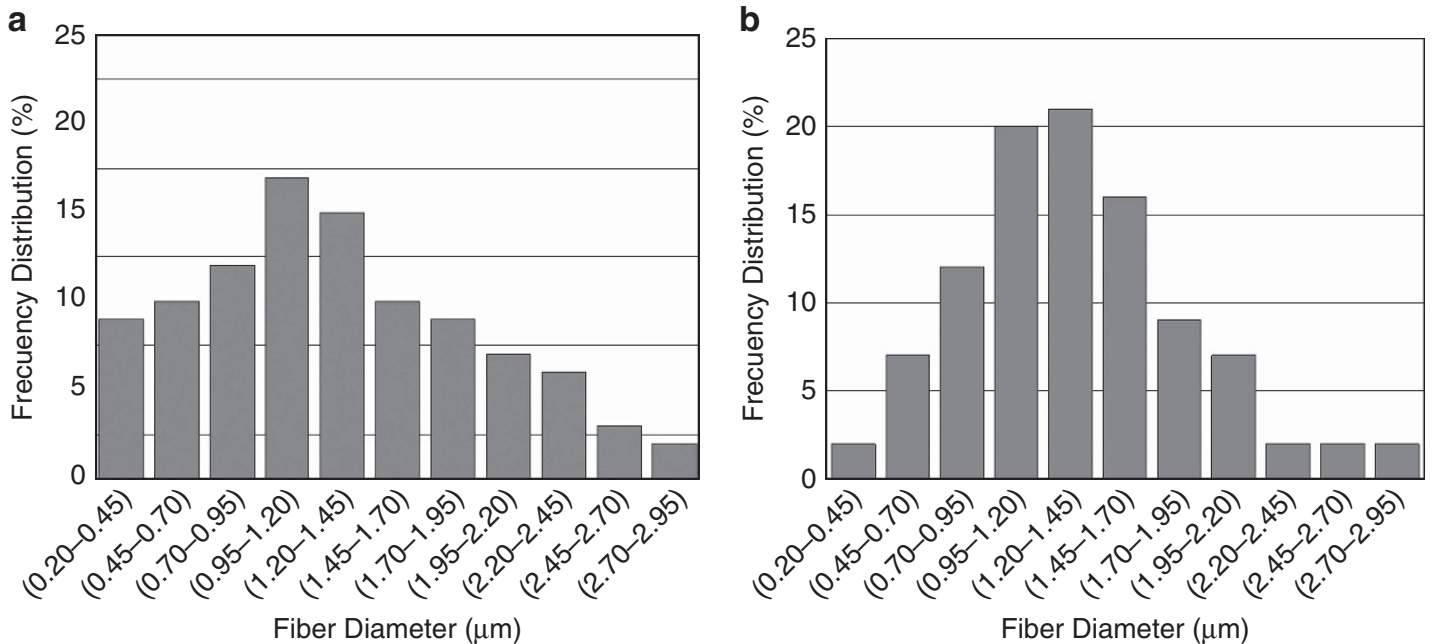

Figure 6 Diameter frequency distribution of electrospun fibers: (a) $P C L_{\mathrm{m}} ; n=100$; diameter: $1.267 \pm 0.576 \mu \mathrm{m}$; (b) $P C L E_{\mathrm{m}} ; n=100$; diameter: $1.286 \pm 0.667 \mu \mathrm{m}$

micrographs (Figure 5). In both cases, the presence of partially crystallized embelin was confirmed later by thermal analysis. Although, in the film, much of the needle-like structures are found at the surface, this morphological feature cannot be directly related to the crystallinity of the samples. In the solution-cast film, the distribution of crystals through the film thickness is not necessarily uniform. However, in the electrospun mesh, the microfibrous structure allowed a better distribution of embelin and its crystalline phase throughout the material.

To study the availability of bioactive agent in microfibrous materials exposed to biological media, the evaluation of device size and geometrical parameters is required. The morphological analysis commonly found in the literature is very simplified and does not take into account the real morphology of the scaffold. A detailed description that includes the device structural aspects is necessary to understand the behavior of drug-loaded, fibrous biomaterials. For this purpose, the effective area was calculated considering the image analysis data. First, the area $A_{i}$ occupied by fibers of a given diameter $D_{i}$ was calculated as follows:

$$
A_{i}=f_{i} \cdot\left(\pi_{i}^{2}\right) / 4 \text {, }
$$

where, $f_{i}$ is the fraction of fibers with a given diameter $D_{i}$, according to data displayed in Figure 6 . The mass occupied by fibers having $D_{i}$ per unit length, $M_{i}$, considering the polymer density $(\rho)$ is

$$
M_{i}=A_{i} \cdot \rho,
$$

and the effective length of the fibers with diameter $D_{i}$ per gram of sample, $L_{i}$ is

$$
L_{i}=1 / M_{i} \text {. }
$$

Then, the total effective area per gram of sample $A_{m e}$, the total effective area per unit volume $A_{v e}$ and the total effective area $A_{T}$ considering both sides of each, were calculated as follows:

$$
\begin{aligned}
& A_{m e}=\sum A_{m i}=\sum\left(L_{i} \cdot \pi \cdot D_{i}\right), \\
& A_{v e}=A_{m e} \cdot \rho, \\
& A_{T}=A_{v e} \cdot V,
\end{aligned}
$$

where, $V$ is the disc volume. Table 1 summarizes the calculated values. These results indicate that $P C L E_{\mathrm{m}}$ samples have a total effective area 1.67-fold higher than that of the PCLE $E_{\mathrm{f}}$ discs. Therefore, the
Table 1 Effective areas $A_{m e}, A_{v e}$ and $A_{T}$ estimated for $P C L_{f}$ and $P C L E_{m}$ discs

\begin{tabular}{lccc}
\hline Sample & $\mathrm{A}_{\mathrm{me}}\left(\mathrm{cm}^{2} \mathrm{~g}^{-1}\right)$ & $\mathrm{A}_{\mathrm{ve}}\left(\mathrm{cm}^{-1}\right)$ & $\mathrm{A}_{\mathrm{T}}\left(\mathrm{cm}^{2}\right)$ \\
\hline$P C L E_{\mathrm{f}}$ & 111.45 & 41.89 & 0.21 \\
$P C L E_{\mathrm{m}}$ & 645.37 & 78.09 & 0.35 \\
\hline
\end{tabular}

Abbreviations: $A_{m e}$, total effective area per gram of sample; $A_{T}$ total effective area; $A_{v e}$, total effective area per unit volume; $P C L_{\mathrm{f}}$, poly( $\varepsilon$-caprolactone) films; $P C L E_{\mathrm{f}}$, embelin-loaded poly( $\varepsilon$-caprolactone) films; $P C L E_{\mathrm{m}}$, embelin-loaded poly( $\varepsilon$-caprolactone) meshes.

morphology of this microfibrous membrane containing highly dispersed embelin assures the higher exposure of embelin to the biological environment. $P C L E_{\mathrm{m}}$ discs also exhibited an increase of $86 \%$ in the effective area-to-volume ratio. Thus, the exposure of embelin could be improved in the PCLE $E_{\mathrm{m}}$ device when compared with $P C L E_{\mathrm{f}}$ because of the fact that the mesh showed a 5.8 -fold increase in the effective area per unit mass.

\section{Embelin quantification}

The embelin content, in terms of the total mass of embelin per disc $\left(m_{\mathrm{EmbT}}\right)$ and the mass of embelin per unit mass of sample $\left(m_{\mathrm{Emb}}\right)$, as determined by ultraviolet-visible spectroscopy, is shown in Table 2 . The obtained data indicate that the loading efficiency of embelin in $P C L E_{\mathrm{m}}$ discs was $83 \%$, whereas in the case of $P C L E_{\mathrm{f}}$ discs, the loading efficiency was $98 \%$. The lower loading efficiency of embelin in the microfibrous meshes can be attributed to electrospinning. During the electrospinning process, the solvent evaporation that occurs in the needle causes the removal of a small amount of embelin that remains attached to the tip, thereby decreasing the amount incorporated in the resulting fibers. The active compound content of the prepared systems is sufficient for the proposed applications.

\section{Embelin release studies}

The release profiles of embelin from $P C L E_{\mathrm{f}}$ and $P C L E_{\mathrm{m}}$ samples are shown in Figure 7. The results show that despite the poor water solubility of embelin, it is released from the hydrophobic PCL matrix into the aqueous solution. Two different stages are seen in the release profile, namely a rapid loss of embelin deposited on the surface of the devices followed by a slow-release phase, probably involving the diffusion of the active agent molecules through the PCL matrix to 
Table 2 Embelin quantification by ultraviolet-visible spectroscopy

\begin{tabular}{lcc}
\hline Sample & $\begin{array}{c}\mathrm{m}_{\text {EmbT }}(\mu \mathrm{g} \text { embelin } \\
\text { per disc) }\end{array}$ & $\begin{array}{c}\mathrm{m}_{\text {Emb }}(\mathrm{mg} \text { embelin } \\
\text { per } \mathrm{g} \text { of sample) }\end{array}$ \\
\hline Film & 93.70 & 46.85 \\
Mesh & 65.07 & 130.15 \\
\hline
\end{tabular}

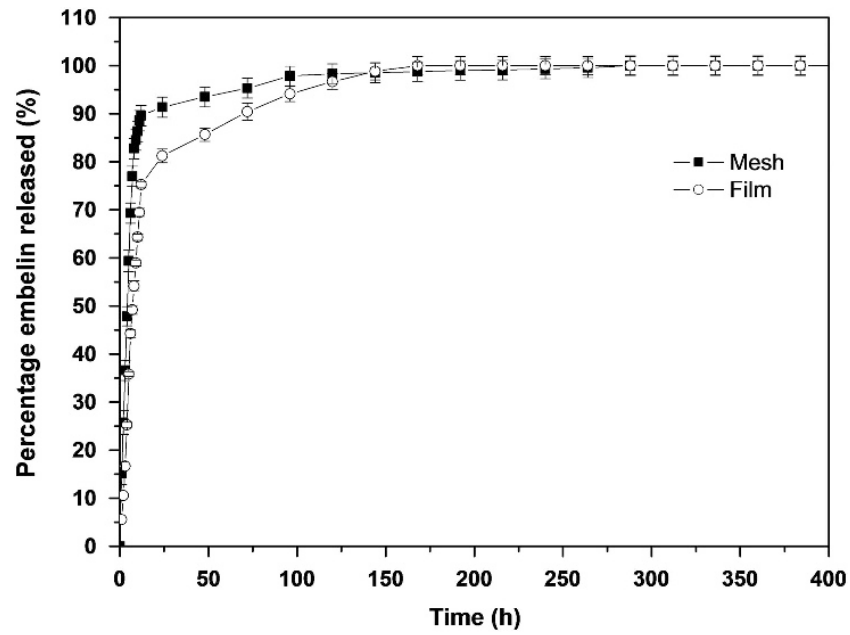

Figure 7 Release profiles of embelin from $P C L E_{f}$ and $P C L E_{\mathrm{m}}$ samples in $\mathrm{PBS}$ at $\mathrm{pH}=7.4$ and $37^{\circ} \mathrm{C}$.

the aqueous solution. The initial burst release observed in both samples lasted $\sim 10 \mathrm{~h}$. However, whereas for the meshes, $80 \%$ of the embelin was already released $\sim 8 \mathrm{~h}$ after exposure to phosphatebuffered saline (PBS), for the films this percentage was reached only after $24 \mathrm{~h}$. Longer periods were required to attain full release, possibly due to the interaction of embelin and PCL. Although the amount of embelin per disc is higher in the films, the mesh had a higher percentage of released embelin owing to its higher effective surface area.

Both types of devices can be considered thin films because of their low thickness values $(0.063 \mathrm{~cm}$ for the mesh and $0.070 \mathrm{~cm}$ for the film) when compared with their diameters (equal to $1 \mathrm{~cm}$ ). Additionally, given that the solubility limit of embelin in PBS at pH 7.4 is $0.13 \mathrm{mg} \mathrm{ml}^{-1}$, according to Patel et al., ${ }^{20}$ it can be assumed that the equilibrium between embelin and the buffer was not reached in either of the tested cases, as the maximum released concentration did not exceed $4 \%$ of the maximum solubility. Therefore, both tested systems remained under sink conditions during the entire period of drugrelease evaluation.

In the present study, the devices in the form of films and meshes can probably both be considered monolithic dispersions because part of the embelin is homogeneously distributed within the PCL matrix at an initial concentration greater than drug solubility. Upon contact with PBS, the solution penetrates into the system and only partially dissolves the drug. As a consequence, dissolved and non-dissolved embelin coexist within the matrix during drug release. Polymer degradation cannot be considered a reason to justify improved drug transport in both types of PCL devices because this polymer has a relatively long hydrolytic degradation time.

Different types of mathematical models are available in the literature to describe the release behavior of drugs incorporated into polymeric devices, as reviewed by Siepmann and Siepmann. ${ }^{26}$ In the simplest case, known as the zero-order release model, the release rate remains constant until the active compound is exhausted in the matrix. If the entire release study period is considered, this is clearly not the behavior observed in any of the evaluated PCL-embelin systems, but this model describes the data from the initial release period for both sets of data reasonably well, probably because of the predominant solid-drug dissolution at this stage.

Another model frequently used and easy to apply is the Peppas equation:

$$
\frac{M_{t}}{M_{\infty}}=k t^{n}
$$

where, $M_{t}$ and $M_{\infty}$ are the cumulative amounts of drug released at time $t$ and infinite time, respectively, $k$ is a constant related to the structural and geometric characteristics of the delivery device and $n$ is the release exponent, which might be indicative of the mechanism of drug release. For thin films, $n$ is equal to 0.5 when Fickian diffusion applies and 1.0 for polymer swelling situations, attaining values between 0.5 and 1.0 in the case of anomalous transport. These models are normally useful for an $M_{t} / M_{\infty}$ ratio lower than 0.6.

When this model was tested to adjust the obtained experimental data in the region of $M_{t} / M_{\infty}<0.6$, values of $R^{2}$ equal to 0.997 and 0.992 and of $n$ equal to 0.85 and 1.14 were determined for the mesh and the film devices, respectively. In the case of the mesh, the superposition of apparently independent mechanisms of drug transport might occur. In all likelihood, this result should be attributed to the rapid dissolution of the solid embelin deposited on the surface of the materials rather than to swelling of the polymer matrix because the PBS absorption by PCL in $10 \mathrm{~h}$ was found to be $<0.5 \%$.

For the film, in addition to the convective transport associated with the dissolution of the solid embelin accumulated at the device surface, because $n>1$, a super-case II transport may be considered, as for instance, a result of void formation in the matrix. ${ }^{27}$ Voids might have formed during casting by rapid volatilization of the solvent within the film, resulting in an increased film surface area and serving as a pathway for the solvent to diffuse through the film. In addition, during embelin release, the dissolution of the active compound might have produced cavities in the PCL matrices, as observed for the antibacterial nalidixic acid incorporated in PCL matrices blended with other biodegradable synthetic polymers. ${ }^{28}$ According to Douglas et al., ${ }^{28}$ when the percentage of active agent incorporated into the polymer matrix is between 5 and $10 \%$, the release mechanism is indeed more complex because the pores resulting from the cumulative exit of the active agent formerly deposited on the surface are filled by the liquid medium, thereby facilitating the release of the compound still incorporated in the polymer matrix. In this situation, as the percentage of incorporated material is relatively low, the pores are frequently not interconnected, but increases in matrix permeability are observed, facilitating drug transport through the system. At higher active agent ratios, the connection of pores might occur, further increasing the permeability of the system.

\section{Thermal properties}

The thermal transitions of free embelin, $P C L_{\mathrm{f}}, P C L_{\mathrm{m}}, P C L E_{\mathrm{f}}$ and $P C L E_{\mathrm{m}}$, were determined by differential scanning calorimetry. The obtained thermograms are shown in Figure 8, whereas the melting temperature $\left(T_{\mathrm{m}}\right)$, the melting heat $\left(\Delta H_{\mathrm{m}}\right)$ and degree of crystallinity $\left(X_{c}\right)$ are summarized in Table 3.

As expected, the solution-cast film $P C L_{\mathrm{f}}$ displayed a higher crystallinity $(49.1 \%)$ compared with the corresponding electrospun 


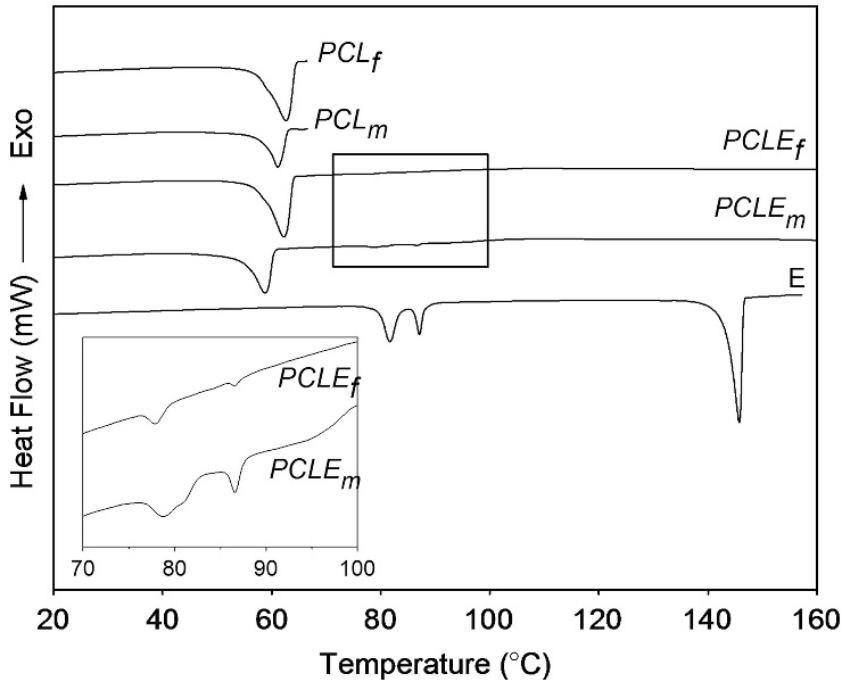

Figure 8 Differential scanning calorimetry thermograms of embelin, $P C L$ and PCLE (films and meshes). Insert: Magnification of signals corresponding to melting of dispersed embelin.

Table 3 Thermal properties of embelin, films and scaffolds

\begin{tabular}{lccccc}
\hline Sample & $\mathrm{T}_{m}\left({ }^{\circ} \mathrm{C}\right)$ & $\Delta \mathrm{H}_{m}\left(\mathrm{Jg}^{-1}\right)$ & $\Delta \mathrm{H}_{m E}\left(\mathrm{Jg}^{-1}\right)$ & $\mathrm{X}_{\mathrm{CPCL}}(\%)$ & $\mathrm{X}_{\mathrm{CE}}(\%)$ \\
\hline$P C L_{\mathrm{f}}$ & 62.6 & 72.70 & - & 49.1 & - \\
$P C L_{\mathrm{m}}$ & 61.1 & 53.05 & - & 35.8 & - \\
Embelin & $81.7,87.1$ & 63.45 & - & - & 100 \\
& 145.8 & 129.68 & & & \\
PCLE $E_{\mathrm{f}}$ & 62.3 & 74.83 & - & 50.5 & - \\
& $77.9,86.5$ & 0.47 & 10.03 & - & 4.7 \\
PCLE & 58.8 & 63.51 & - & 42.9 & - \\
& $79.0,86.6$ & 2.16 & 16.60 & - & 13.0 \\
\hline
\end{tabular}

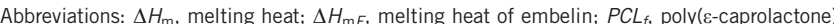
films; $P C L_{m}$, poly( $\varepsilon$-caprolactone) meshes; $P C L E_{f}$, embelin-loaded poly( $\varepsilon$-caprolactone) films; $P C L E_{\mathrm{m}}$, embelin-loaded poly( $\varepsilon$-caprolactone) meshes; $T_{\mathrm{m}}$, melting temperature; $X_{C P C L}$, degree of crystallinity of PCL; $X_{C E}$, degree of crystallinity of embelin.

mesh $P C L_{\mathrm{m}}(35.8 \%)$. The same observation for PCL nanofibrous meshes was performed by Lee et al. ${ }^{29}$ after analyzing wide angle X-ray diffraction patterns. Structure formation during electrospinning is governed by the simultaneous processes of solvent evaporation and elongation of the solidifying fiber. In semicrystalline polymers, solidification is also concurrent with crystal nucleation. The shorter time for crystallization is expected to limit the development of crystallinity, resulting in small crystallites with defects and thus a lower degree of crystallinity. ${ }^{30}$ In the case of PCL, the crystallization process could not be completely suppressed.

In the case of drug-loaded samples, the crystallinity of PCL also decreased in the electrospun samples, probably facilitating drug transport within the matrix after exposure to PBS. Embelin displayed three well-defined melting peaks. However, these melting signals are almost completely absent in the embelin-loaded samples $P C L E_{\mathrm{f}}$ and $P C L E_{\mathrm{m}}$ (see insert in Figure 8). Embelin showed a strong reduction in the degree of crystallinity (from 100 to $4.7 \%$ in the film and $13 \%$ in the mesh), indicating a marked solubilization of embelin in the PCL matrix. The crystallization of embelin not only could not be fully circumvented but also showed an opposite behavior in comparison with the polymer, increasing more in the electrospun samples. From these results, it is clear that the electrospinning process affects the polymer and the drug in different ways. This behavior can be explained by the different structure and chain length of these species that could allow the formation of a small fraction of embelin crystals during jet formation. The polymer carrier stabilized the formed amorphous drug dispersion/solution through vitrification or via specific molecular interactions, with the tendency for the drug to recrystallize being suppressed or at least made kinetically unfavorable for a long enough period to make the material pharmaceutically useful. $^{31}$

\section{CONCLUSIONS}

Poly( $\varepsilon$-caprolactone) matrices containing embelin were successfully obtained by solution casting and electrospinning. The bioactive agent was significantly dissolved in the polymeric matrices, as shown by the thermal analysis. Microfibrous, embelin-loaded PCL meshes exhibited a significant decrease in drug crystallinity, indicating good solubilization in the polymer matrix. Moreover, the improved capacity of the meshes to expose amorphous embelin as well as the higher surface area-to-volume and mass-to-volume ratio make the porous scaffolds appropriate for preparing drug-loaded, polymeric matrices with improved efficiency if a relatively high drug-release rate is desired. The in vitro and in vivo antifungal efficiencies of embelin-loaded meshes against dermatophytes, such as Trichophyton mentagrophytes, are currently under investigation.

\section{CONFLICT OF INTEREST}

The authors declare no conflict of interest.

\section{ACKNOWLEDGEMENTS}

We are grateful to CICITCA-UNSJ and Departamento de Electrónica y Automática (FI UNSJ). PRCT thanks the CONICET and ÂMM thanks the National Council for Scientific and Technological Development (CNPq/Brazil) for the fellowships awarded. We thank Dra MA González for her useful suggestions regarding image processing. This work was supported by the Argentinean National Agency of Scientific and Technological Promotion (PICT 448 grant) and the National Research Council (PIP 522 Grant). The RIMADEL network funded by CYTED is also acknowledged.

1 Leuner, C. \& Dressman, J. Improving drug solubility for oral delivery using solid dispersion. Eur. J. Pharm. Biopharm. 50, 47-60 (2000).

2 Serajuddin, A. T. Solid dispersion of poorly water-soluble drug: early promises, subsequent problems and recent breakthroughs. J. Pharm. Sci. 88, 1058-1066 (1999).

3 Brewster, M. E., Verreck, G., Chun, I., Rosenblatt, J., Mensch, J., Van Dijck, A., Noppe, M., Ariën, A., Bruning, M. \& Peeters, J. The use of polymer-based electrospun nanofibers containing amorphous drug dispersions for the delivery of poorly watersoluble pharmaceuticals. Pharmazie 59, 387-391 (2004).

4 Verreck, G., Chun, I., Peeters, J., Rosenblatt, J. \& Brewster, M. E. Preparation and characterization of nanofibers containing amorphous drug dispersions generated by electrostatic spinning. Pharm. Res. 20, 810-817 (2003).

5 Sill, T. J. \& Von Recum, H. A. Electrospinning: applications in drug delivery and tissue engineering. Biomaterials 29, 1989-2006 (2008).

6 Bhardwaj, N. \& Kundu, S. C. Electrospinning: a fascinating fiber fabrication technique. Biotech. Adv. 28, 325-347 (2010).

7 Kenawy, E. R., Abdel-Hay, F. I., El-Newehy, M. H. \& Wnek, G. E. Processing of polyme nanofibers through electrospinning as drug delivery systems. Mat. Chem. Phys. 113, 296-302 (2009).

8 Chakraborty, S., Liao, I.-C., Adler, A. \& Leong, K. W. Electrohydrodynamics: a facile technique to fabricate drug delivery systems. Adv. Drug Del. Rev. 61, 1043-1054 (2009).

9 Greiner, A. \& Wendorff, J. H. Electrospinning: a fascinating method for the preparation of ultrathin fibers. Angew. Chem. Int. Ed. 46, 2-36 (2007).

$10 \mathrm{Li}$, D. \& Xia, Y. Electrospinning of nanofibers: reinventing the wheel? Adv. Mater. 16 $1151-1170$ (2004).

11 Albertsson, A. C. \& Varma, I. K. Aliphatic polyesters: synthesis, properties and applications. Adv. Polym. Sci. 157, 1-40 (2002). 
12 Haq, K., Ali, M. \& Siddiqui, A. W. New compounds from the seeds of Embelia ribes burm. Pharmazie 60, 69-71 (2005).

13 Raja, S., Unnikrishnan, K. P., Ravindran, P. N. \& Balachandran, I. Determination of embelin in Embelia ribes and Embelia tsjeriam-cottam by HPLC. Indian J. Pharm. Sci. 67, 734-736 (2005).

14 Feresin, G. E., Tapia, A., Sortino, M., Zacchino, S., de Arias, A. R., Inchausti, A., Yaluff, G., Rodriguez, J., Theoduloz, C. \& Schmeda-Hirschmann, G. Bioactive alkyl phenols and embelin from Oxalis erythrorhiza. J. Ethnopharmacol. 88, 241-247 (2003).

15 Sreepriya, M. \& Bali, G. Chemopreventive effects of embelin and curcumin against $\mathrm{N}$-nitrosodiethylamine/phenobarbital-induced hepatocarcinogenesis in Wistar rats. Fitoterapia 76, 549-555 (2005).

16 Wango, E. O. Anti-fertility effects of embelin in female Sprague-Dawley rats may be due to suppression of ovarian function. Acta. Biol. Hung. 56, 1-9 (2005).

17 Podolak, I., Galanty, A. \& Janeczko, Z. Cytotoxic activity of embelin from Lysimachia punctata. Fitoterapia 76, 333-335 (2005).

18 Mahendran, S., Thippeswamy, B. S. \& Veerapur, V. P. Anticonvulsant activity of embelin isolated from Embelia ribes. Phytomedicine 18, 186-188 (2011).

19 Mahendran, S., Badami, S. \& Maithili, V. Evaluation of antidiabetic effect of embelin from Embelia ribes in alloxan induced diabetes in rats. Biomed. Pharmacother. (doi:10.1016/j.biopha.2010.08.003).

20 Patel, R. K., Pundarikakshudu, K., Momin, M. \& Patel, M. M. Studies on formulation and in vitro dissolution of embelin tablets. Indian J. Pharm. Sci. 68, 227-230 (2006).

21 Chitra, M., Sukumar, E., Suja, V. \& Devi, C. S. Antitumor, anti-inflammatory and analgesic property of embelin, a plant product. Chemother. 40, 109-113 (1994).
22 Radhakrishnan, N., Gnanamani, A. \& Mandal, A. B. A potential antibacterial agent embelin, a natural benzoquinone extracted from Embelia ribes. Biol. Med. 3, 1-7 (2011).

23 Van Krevelen, D. W. Properties of Polymers. 3rd edn, Vol. 121 (Elsevier, Amsterdam, 1990).

24 Renecker, D. H. \& Chun, I. Nanometre diameter fibres of polymer, produced by electrospinning. J. Nanotech. 7, 216-223 (1996).

25 Gonzalez, M. A., Montini Ballarin, F., Brun, M., Abraham, G. A. \& Ballarin, V. Morphological quantification of polymer nanofibers in tissue engineering images. L.A.A.R 42, 89-95 (2012).

26 Siepmann, J. \& Siepmann, F. Mathematical modeling of drug delivery. Int. J. Pharmaceut. 364, 328-343 (2008).

27 Barr-Howell, B. D. \& Gordon, E. J. A study of the transport properties of solvent desorption from experimental films using thermogravimetric analysis. Thermochim. Acta. 180, 147-154 (1991).

28 Douglas, P., Andrews, G., Jones, D. \& Walker, G. Analysis of in vitro drug dissolution from PCL melt extrusion. Chem. Eng. J. 164, 359-370 (2010).

29 Lee, K. H., Kim, H. Y., Khil, M. S., Ra, Y. M. \& Lee, D. R. Characterization of nanostructured poly( $\varepsilon$-caprolactone) nonwoven mats via electrospinning. Polymer 44, 1287-1294 (2003).

30 Zong, X., Kim, K., Fang, D., Ran, S., Hsiao, B. S. \& Chu, B. Structure and process relationship of electrospun bioabsorbable nanofiber membranes. Polymer 43, 4403-4412 (2002).

31 Leuner, C. \& Dressman, J. Improving drug solubility for oral delivery using solid dispersion. Eur. J. Pharm. Biopharm. 50, 47-60 (2000). 